Nephrologe 2016 $11: 382$

DOI 10.1007/s11560-016-0106-x

Online publiziert: 7. November 2016

(c) Springer-Verlag Berlin Heidelberg 2016

CrossMark

\author{
U. Heemann' $\cdot$ 0. Witzke ${ }^{2}$ \\ ${ }^{1}$ Klinik und Poliklinik für Innere Medizin II, Abteilung für Nephrologie, Klinikum rechts der Isar, TU \\ München, München, Deutschland \\ ${ }^{2}$ Klinik für Infektiologie, Zentrum für Innere Medizin, Westdeutsches Zentrum für Infektiologie, Innere \\ Medizin \& Nephrologie/Infektiologe/Hypertensiologe DHL, Universitätsklinikum Essen, Essen, \\ Deutschland
}

\title{
Infektionen und Nephrologie
}

Eine chronische Nierenerkrankung wirkt sich auf viele Systeme des Körpers aus. Unter anderem führt der partielle Ausfall der Nierenfunktion zu Störungen der angeborenen und der adaptiven Immunantwort, sodass Infektionen in dieser Patientengruppe die zweithäufigste Todesursache sind. Weiterhin hat in der $\mathrm{Ne}$ phrologie die immunsuppressive Therapie einen wesentlichen Stellenwert in der Behandlung von Autoimmunerkrankungen (z. B. Glomerulonephritiden, Kollagenosen, Vaskulitiden), vor allem aber im Rahmen der Nierentransplantation. Die Weiterentwicklung der immunsuppressiven Therapie hat in der Nephrologie entscheidende therapeutische Fortschritte möglich gemacht. Der Kehrseite dieser effektiven und $z$. T. sehr intensiven Immunsuppression ist eine erhöhte Anfälligkeit gegenüber Krankheitserregern und Malignomen.

Das Auftreten von Infektionen bestimmt in hohem Maße die Morbidität, und erschwerend kommt hinzu, dass immunsupprimierte Patienten aufgrund häufiger Krankenhausaufenthalte und/ oder antibiotischer Vortherapien oft mit multiresistenten Erregern (MRE) kolonisiert sind, was bei der Wahl des Therapieregimes bedacht werden muss. Erregergruppen, die dabei eine zunehmende Herausforderung darstellen, sind vancomycinresistente Enterokokken (VRE), methicillinresistente Staphylococcus aureus und multiresistente gramnegative (MRGN) Erreger (Enterobakterien, Pseudomonas aeruginosa, Klebsiella pneumoniae etc.). Patienten mit chronischer Nierenkrankheit und nach Nierentransplantation haben ein erhöhtes Risiko für eine MRE-assoziierte Mortalität und Morbidität. Nicht zwin- gend ist das Auftreten von Fieber durch eine Infektion verursacht - differenzialdiagnostisch müssen Erkrankungen wie hereditäre Fiebersyndrome beachtet werden. Nephrologen sind ohnehin mit diesen Patienten konfrontiert, da diese zum größten Teil auch an einer Nierenkrankheit leiden.

Im vorliegenden $\mathrm{Heft}$ von $\mathrm{Der} \mathrm{Ne}$ phrologe sind nephrologische Fragestellungen bei speziellen Infektionen und bei der BK-Virus-Infektion nach Organtransplantation sowie renale Manifestationen bei der HIV-Infektion beschrieben. Daneben wird ein Überblick über die hereditären Fiebersyndrome und Infektionen unter Immunsuppression gegeben. Die Diagnose und die Therapie von Infektionen bei immunsupprimierten Patienten stellen eine Herausforderung für den behandelnden Arzt dar.

Wegen der Vielfalt und der Bedeutung infektiologischer Fragestellungen bei nephrologischen Patienten sollte das Erwerben infektiologischer Kenntnisse zur Basis der Ausbildung zum Nephrologen gezählt werden. Wir hoffen, mit dem beiliegenden Heft dazu einen Beitrag leisten zu können.

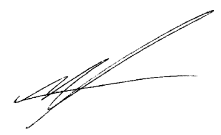

U. Heemann

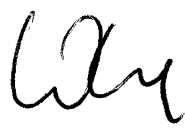

O. Witzke

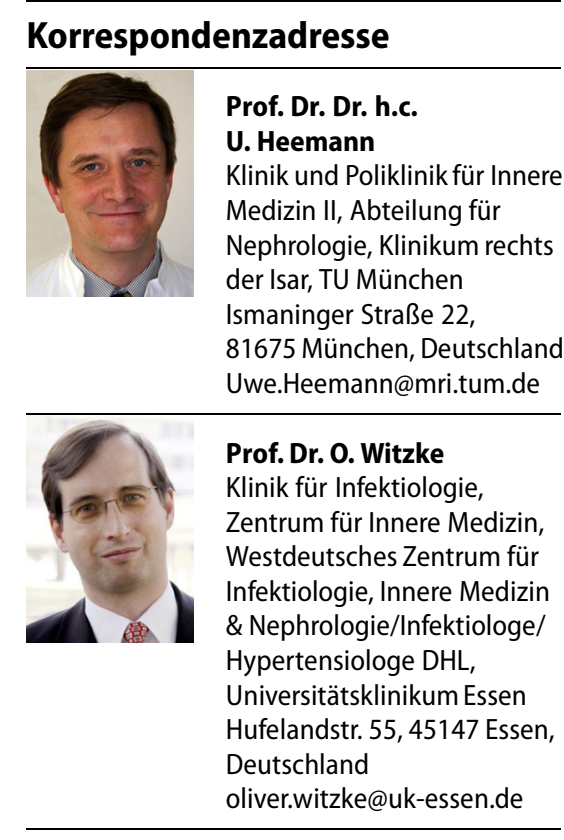

Interessenkonflikt. U. Heemann und O. Witzke geben an, dass kein Interessenkonflikt besteht. 